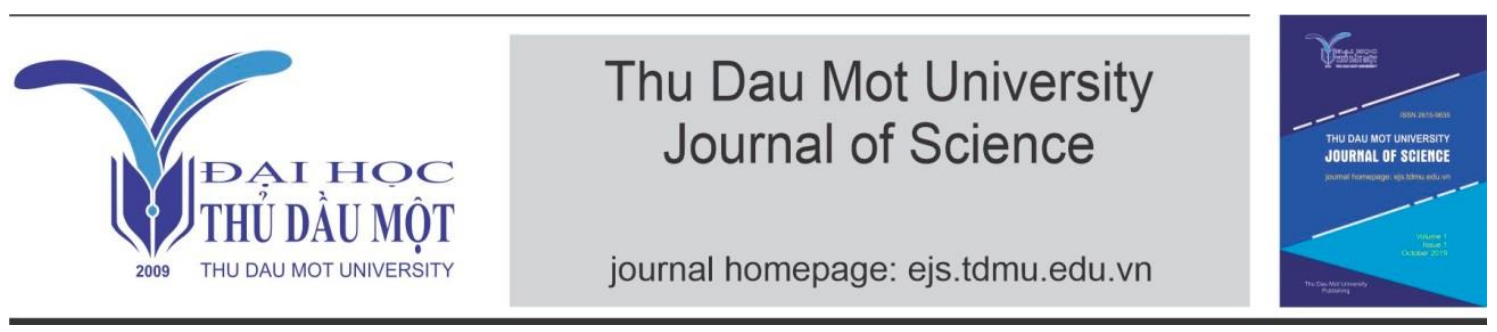

\title{
Entropy Measurement to Extract the Signification of Abnormal Activity from Camera's Frames and its Application for Fall Detection
}

by Hoang Manh Ha, Tran Ba Minh Son, Nguyen Xuan Dung (Thu Dau Mot University), Vo Quoc Thong (Binh Duong General Hospital)

Article Info: Received 20 Jun 2020, Accepted 22 Oct 2020, Available online 15 Dec, 2020 Corresponding author: hahm@tdmu.edu.vn

https://doi.org/10.37550/tdmu.EJS/2020.04.081

\begin{abstract}
Most of the indoor accidents are related with fall down. Many medical studies are point out that key factor for keeping patient's life is fast response of monitoring system. In modern life, peoples are isolated with neighbor, especially in living quarters. Therefore many solutions are developed for falling down monitoring that base on wearable sensors. These methods require of an expensive sensors system with electric power supplier and telecommunication devices. In context of patients with disease and weak status, patients are trend to remove sensor system. This issue requires to find out another approach so that sensors system will not be needed. We study the fall detection by monitoring camera. For increase the accuracy, we proposed a simple and effective method to extract features of abnormal activities. By tracking the magnitude of entropy and its distribution, our fall detection model has a capability of differentiating falls from other activities
\end{abstract}

Keywords: feature extraction, fall monitoring, chaos of information, entropy

\section{Introduction}

A development of smart cities has motivated for many higher request in living. The life quality of elder is most expected factor. Therefore, a fall monitoring by camera is one of important problems. Aggarwal (2011) shows that an abnormal activity is strongly 
Hoang Manh Ha, Vo Quoc Thong,... - Volume 2- Issue 4-2020, p.363-372.

related to fall in elder [1]. This was motivation for studying the abnormal activity detection. In the literature, researchers proposed different methods [1-8, 11-19] to detect abnormal activity, researches focus on bellowing approaches. The abnormal activities detection techniques is briefly summarized in below table

TABLE 1. Summarization of methods for activities recognition

\begin{tabular}{|c|c|c|c|}
\hline No & Main author & Description & Reference \\
\hline 1 & Khalid. S & $\begin{array}{l}\text { The template matching method based on the similarity } \\
\text { between activities that pre-determine. In fact, this method } \\
\text { highly probability generate a fail negative result when fall } \\
\text { be happened in new way, Khalid. S generalized this } \\
\text { problem by statistic aspect, in [2] he shown that a fall } \\
\text { activity uncorrelated to normal activity. }\end{array}$ & {$[2]$} \\
\hline 2 & Yin, J.; Meng, Y & \multirow{3}{*}{$\begin{array}{l}\text { In method of state space, a normal activity is } \\
\text { formulated in a statistical model by training. An } \\
\text { abnormal activity is detected by deviation from } \\
\text { statistical parameter of normal activity. }\end{array}$} & [3] \\
\hline 3 & $\begin{array}{l}\text { Loy, C.C. Xiang, T. } \\
\text { Gong, S }\end{array}$ & & [4] \\
\hline 4 & Hu, D.H.; Yang, Q & & [5] \\
\hline 5 & $\begin{array}{l}\text { Lui, Y. Beveridge, J.R. } \\
\text { Kirby, M }\end{array}$ & \multirow{2}{*}{$\begin{array}{l}\text { Manifolds Geometry method is based on the relation } \\
\text { between human activities and particular matrix } \\
\text { manifold }\end{array}$} & [6] \\
\hline 6 & Lui, Y & & [7] \\
\hline 7 & $\begin{array}{l}\text { Anice Jahanjoo, Marjan } \\
\text { Naderan and Mohammad } \\
\text { Javad Rashti }\end{array}$ & $\begin{array}{l}\text { Classify abnormal activities by deep belief network } \\
\text { algorithms }\end{array}$ & {$[8]$} \\
\hline 8 & $\begin{array}{l}\text { O. Popoola and } \mathrm{K} . \\
\text { Wang }\end{array}$ & The abnormal activities is defined by training data & {$[11]$} \\
\hline 9 & $\begin{array}{l}\text { G. J. Burghouts, V. P. } \\
\text { Slingerland, H. ten } \\
\text { R.J.M, H. den R.J.M, } \\
\text { and K. Schutte }\end{array}$ & $\begin{array}{l}\text { the irregularities is descripted by expert in action } \\
\text { monitoring }\end{array}$ & {$[12]$} \\
\hline 10 & $\begin{array}{l}\text { H.Nallaivarothayan, C. } \\
\text { Fookes, S. Denman, } \\
\text { and S. Sridharan }\end{array}$ & Action monitor using un-supervisor learning & [13] \\
\hline \multirow[t]{2}{*}{11} & $\begin{array}{l}\text { Y. Benabbas, N. } \\
\text { Ihaddadene, and C. } \\
\text { Djeraba }\end{array}$ & \multirow{3}{*}{ The abnormal activities detected by clustering } & {$[14]$} \\
\hline & $\begin{array}{l}\text { C. Piciarelli and G. L. } \\
\text { Foresti }\end{array}$ & & {$[15]$} \\
\hline 12 & B. Antic and B. Ommer & & {$[16]$} \\
\hline 13 & \begin{tabular}{l|} 
A. Adam, E. Rivlin, I. \\
Shimshoni and D. Reinitz
\end{tabular} & $\begin{array}{l}\text { The abnormal activities is recognized by the difference } \\
\text { in velocity and trend }\end{array}$ & {$[17]$} \\
\hline 14 & $\begin{array}{l}\text { M. Roshtkhari and M. } \\
\text { Levine }\end{array}$ & \multirow[b]{2}{*}{ Base on pixels } & {$[18]$} \\
\hline 15 & $\begin{array}{l}\text { V. Mahadevan, W. Li, } \\
\text { V. Bhalodia, and N. } \\
\text { Vasconcelos }\end{array}$ & & [19] \\
\hline
\end{tabular}




\section{Proposed method}

Teng Li presented in [10] that the features extraction is directly effecting to accuracy of the results.

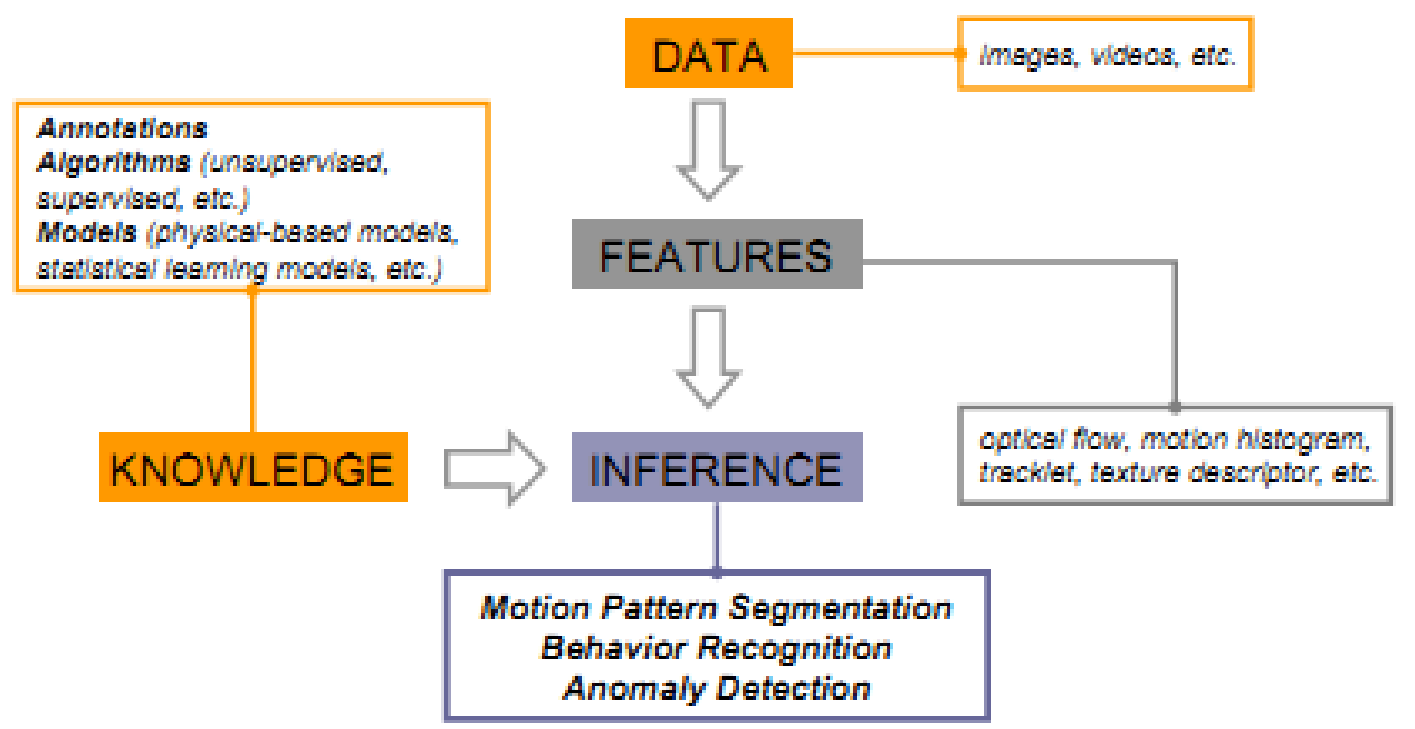

Figure 1. Scheme of the recognized system [10]

Therefore he most important work for activities recognizing is features extraction. This paper focus on a proposes that to give a method for extraction signification of abnormal activities that allow to automatically detect fall in elder that would typically require a human supervisor

The key contribution of our study is applying Entropy measurement to highlight the features of abnormal activity.

Entropy measurement

Entropy measurement can be mathematically defined as

$$
H=-K \sum_{i=1}^{n} p_{i} \log p_{i}
$$

where

$K$ is a positive constant.

$p_{i}$ is a probability of event $i$

Shannon shown that the entropy measurement has a relative to magnitude of a chaos of the information [9]. The chaos of information is highly related to abnormal activity. This idea gave us inspire to solve aboved problem, extract features of the abnormal activity. 
Hoang Manh Ha, Vo Quoc Thong,... - Volume 2- Issue 4-2020, p.363-372.

The chaos at pixel with row $\mathrm{y} 0$ and column $\mathrm{x} 0$ is estimated as bellowed description

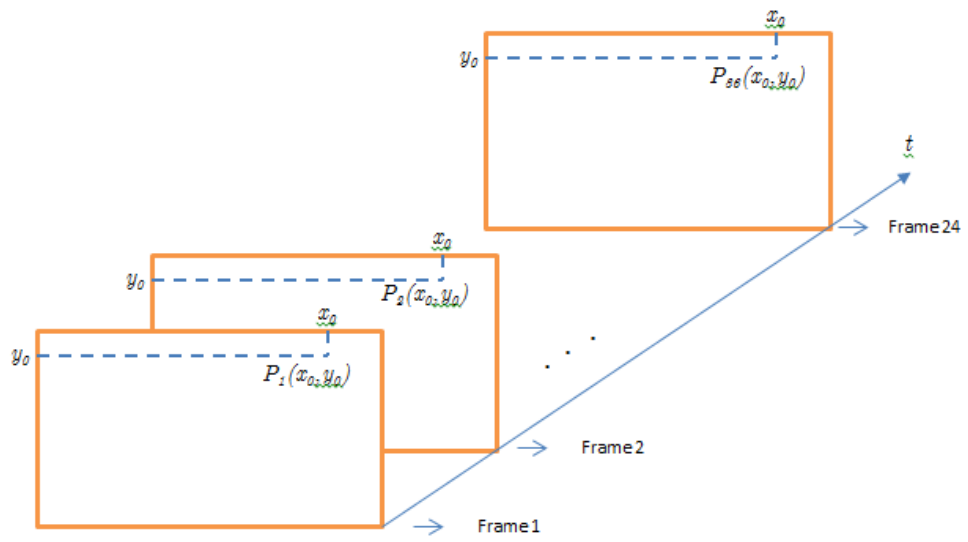

Figure 2. Description of entropy estimation at pixel with row $y_{0}$ and column $x_{0}$ over 24 frames

Proposed a method to estimate the chaos at each pixel through magnitude of entropy

At pixel $P\left(x_{0}, y_{0}\right)$ we denote that,

$P 1\left(x_{0}, y_{0}\right)$ is value of pixel $P 1$ that belong frame no 1 . Similar,

$P 2\left(x_{0}, y_{0}\right)$ is value of pixel $P 2$ that belong frame no 2 .

$P 24\left(x_{0}, y_{0}\right)$ is value of pixel $P 24$ that belong frame no 24 .

$H\left(x_{0}, y_{0}\right)$ is magnitude of entropy at pixel $P l\left(x_{0}, y_{0}\right)$ over 24 frames. $H\left(x_{0}, y_{0}\right)$ is computed by entropy function of Matlab.

Illustrate the relation between abnormal activity and entropy

Fig 3 illustrate from frame 1 to 24, with fall man at central of room
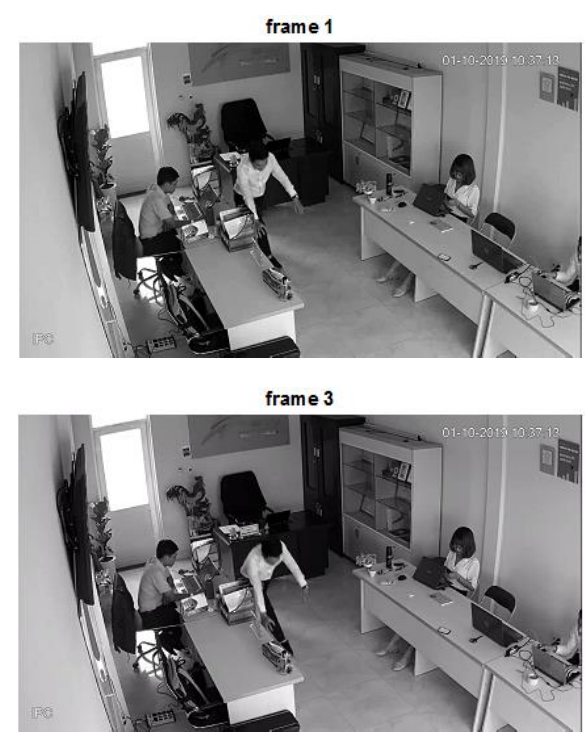
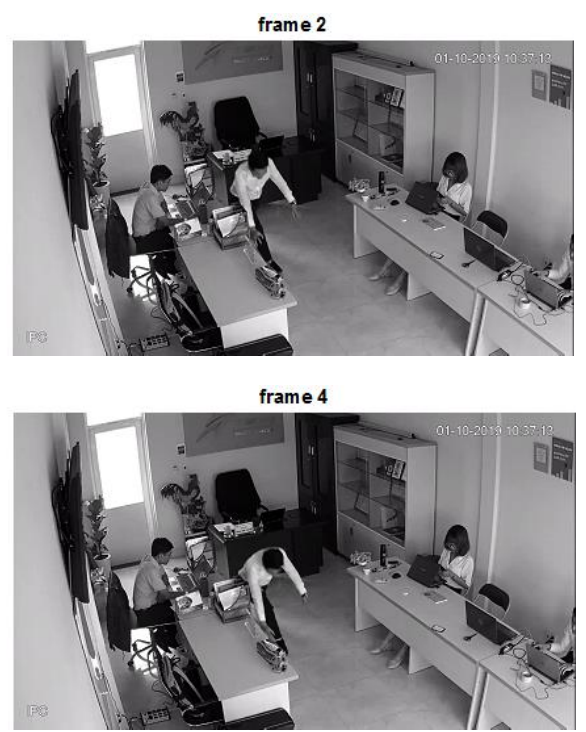

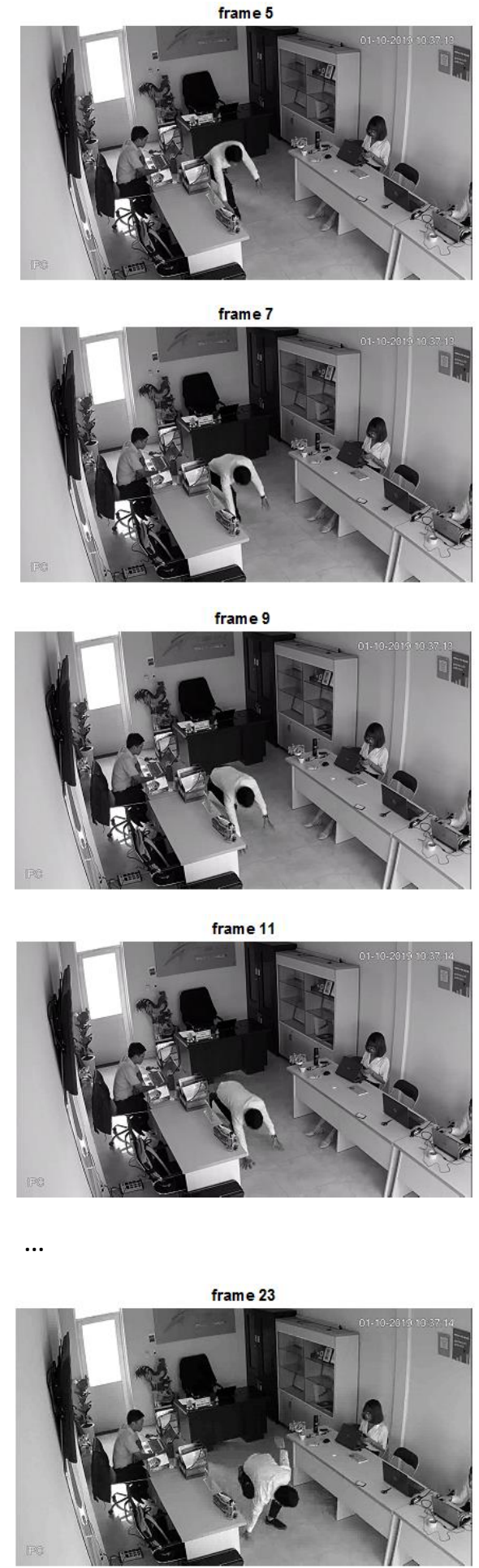
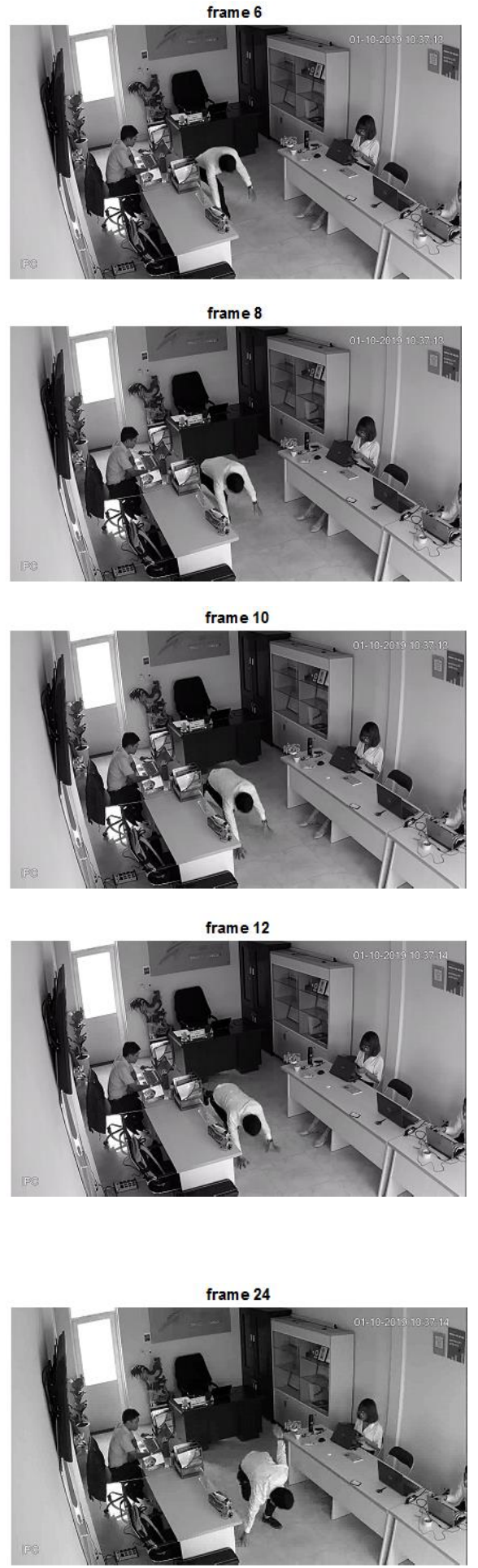

Figure 3. Frames 18 to 24

We estimated entropy for all pixels over 24 frames and the result is shown in fig 4. In fig 3, domain with black dots where reflected entropy close to zero. The segments without black dots reflected that entropy larger than zero that mean exist some chaos as fall. 
Hoang Manh Ha, Vo Quoc Thong,... - Volume 2- Issue 4-2020, p.363-372.

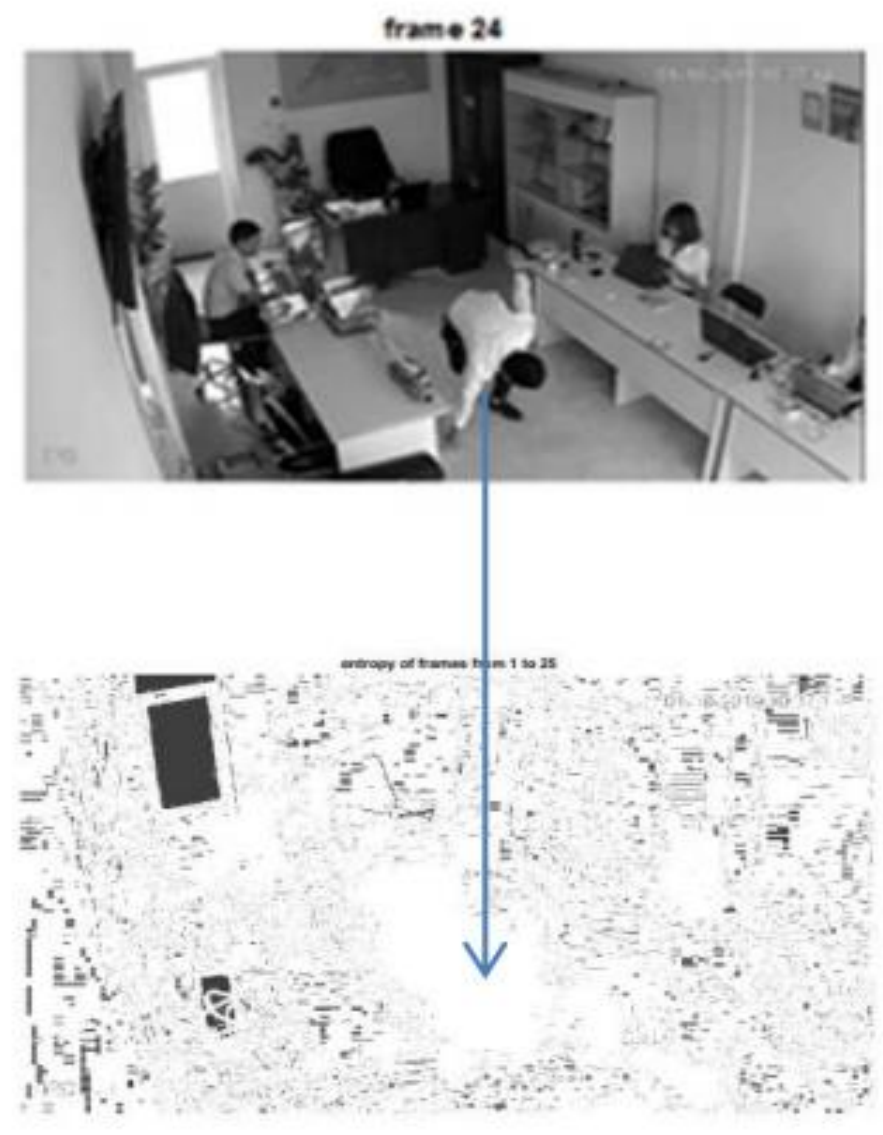

Figure 4. Entropy of frame 1 to 24.

Figure 5 illustrated an opposite cases, without fallen man.
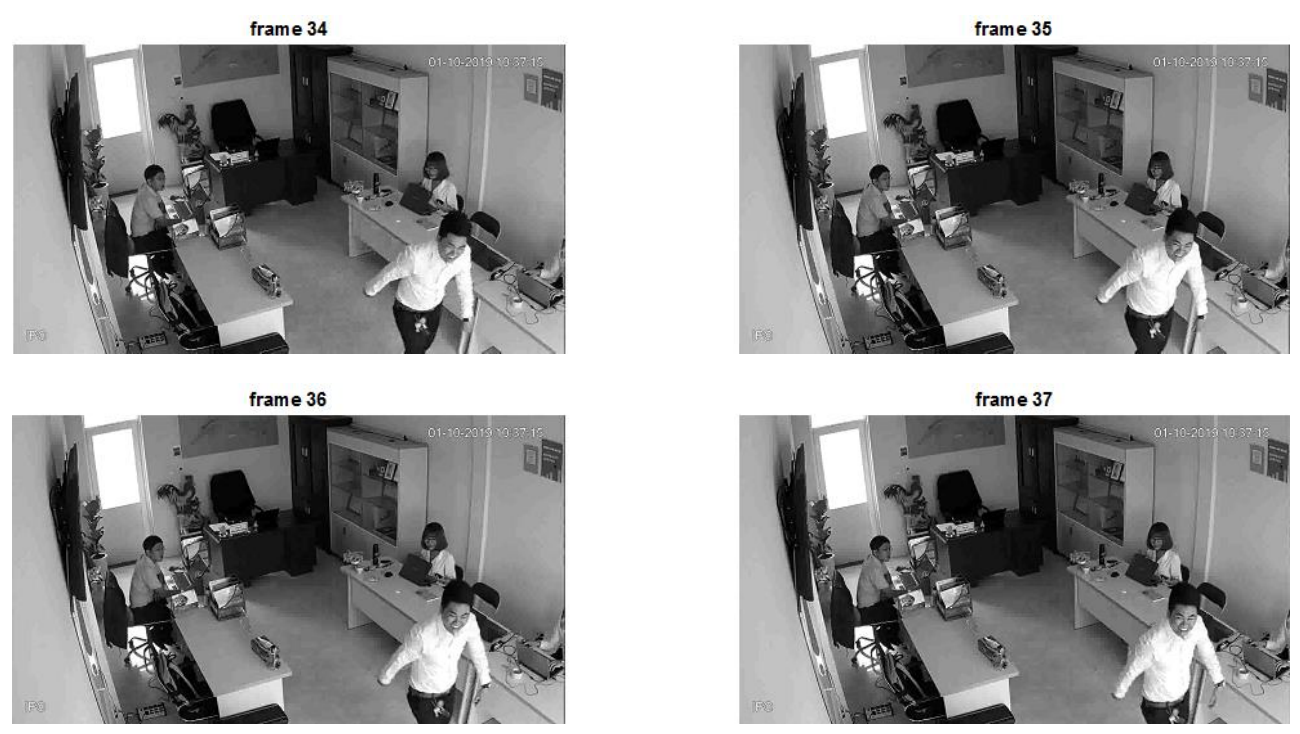

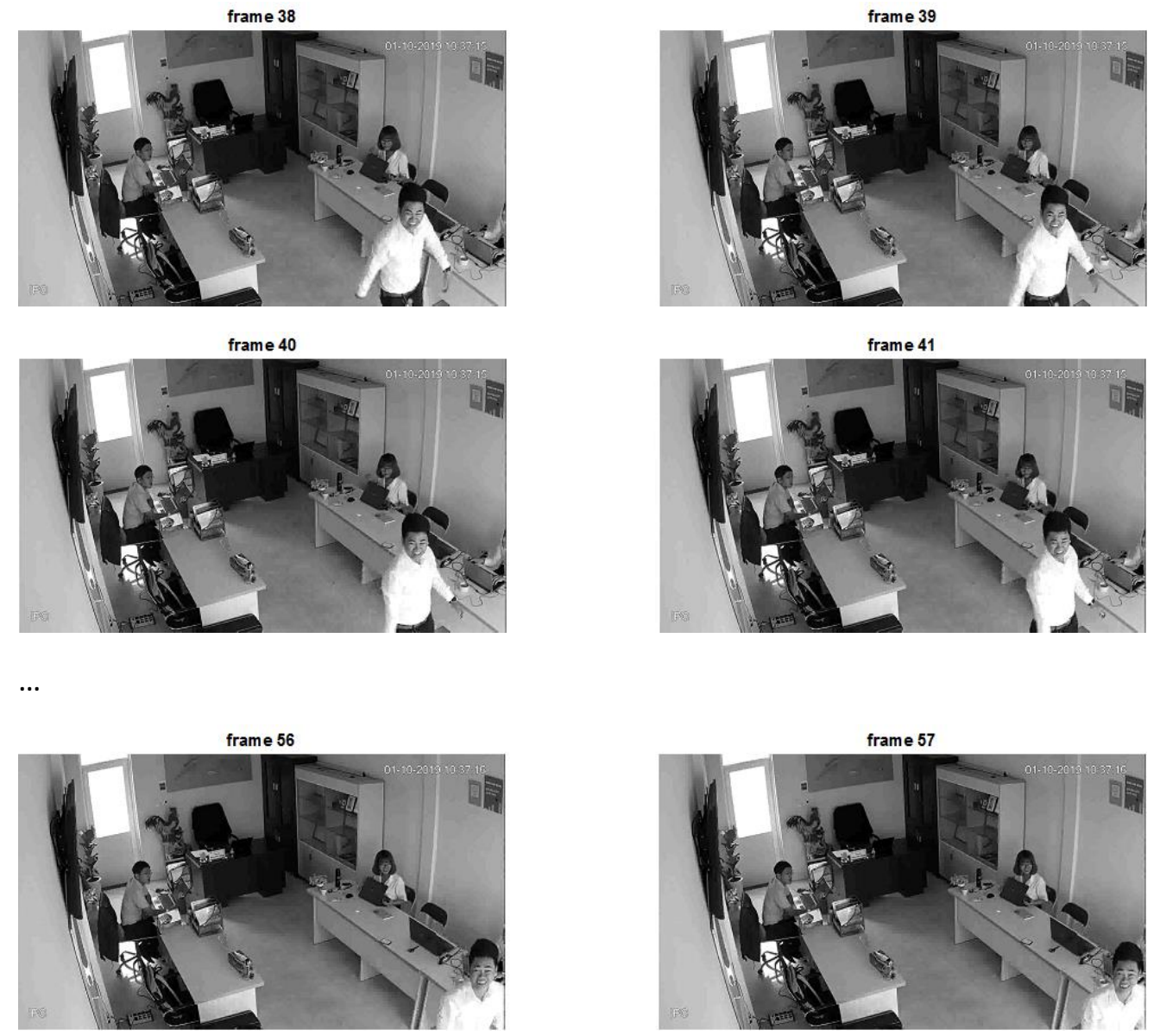

Figure 5. Frames 34 to 57.

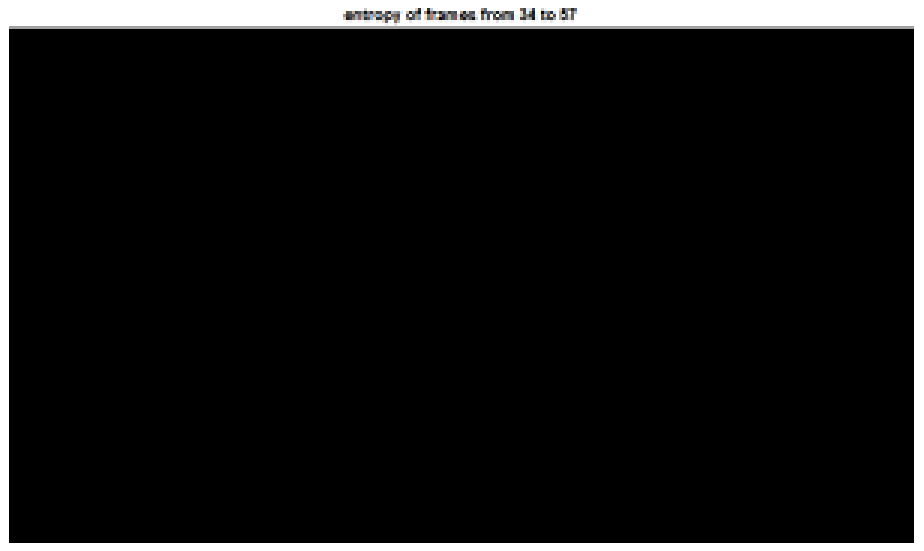

Figure 6. Entropy of frames 34 to 57.

By another word, this research visualized by entropy measurement so that almost elements of $2 \mathrm{D}$ array are close to zero whenever falling is not happen. This is particularly meaningful for classification purposes 
Hoang Manh Ha, Vo Quoc Thong,... - Volume 2- Issue 4-2020, p.363-372.

\section{Experiment}

Our method was implemented using matlab R2016a, on a PC using Intel dual core 2.0 GHz CPU, with 8GB RAM. In this article, we introduce an application of our proposed method that is fall man detection. MATLAB statistic toolbox is used for supporting pratical results in this paper.

Dataset for action recognition contains 2 activities, such as falling action and running (without falling). The collection of data was implemented by us.

The no of forgeries accepted by the system are given as the FAR that is False Acceptance Ratio which is measured as the ratio of no. of forgeries accepted to no. of forgeries considered for evaluation. So, FAR is calculated by the followed formula

$$
F A R=\frac{N_{f a}}{N_{f t}} 100
$$

where $N_{f a}$ is number of forgeries accepted and $N_{f t}$ is number of forgeries tested.

The no of originals rejected by the system are given as the FRR that is False Rejection Ratio which is measured as the ratio of no. of originals rejected to no. of original signatures considered for evaluation. So, FRR is calculated by the formula given in equation

$$
F R R=\frac{N_{o r}}{N_{o t}} 100
$$

where $N_{o r}$ is number of originals rejected and $N_{o t}$ is number of originals tested.

TABLE 2. Experimental results and evaluation of our approach

\begin{tabular}{lll}
\hline Number of samples & FAR $(\%)$ & FRR $(\%)$ \\
\hline Clip1 & 2.5 & 5.0 \\
Clip2 & 1.5 & 6.2 \\
Clip3 & 1.2 & 4.5 \\
Clip4 & 1.5 & 5.5 \\
Clip5 & 2.2 & 6.5
\end{tabular}

TABLE 3. Comparison of detection techniques

\begin{tabular}{lll}
\hline Reference & Dataset & Accu (\%) \\
\hline Benabbas [14] & CUHK & 77 \\
Mahadevan [19] & UCSD & 75 \\
Our method & CUHK & 92.3
\end{tabular}




\section{Conclusion}

In this research paper, the entropy based for fall Monitoring is presented to save lives and property damages. The objective of this paper is to detect fall man by improvement the quality of features. The performance evaluation need more samples clip for its implement.

\section{References}

[1] Aggarwal. J.K, Ryoo. M.S (2011), Human activity analysis: A review. ACM Comput. Surv , 43, art no. 16

[2] Khalid, S. Naftel (2005), A. Classifying spatiotemporal object trajectories using unsupervised learning of basis function coefficients. In Proceedings of the 3rd ACM International Workshop on Video Surveillance \& Sensor Networks, New York, NY, USA, $1-2$, pp. $45-52$.

[3] Yin, J.; Meng, Y (2009), Abnormal behavior recognition using self-adaptive hidden markov models. Lect. Notes Comput. Sci, 5627, 337-346.

[4] Loy, C.C.; Xiang, T.; Gong, S (2009), Surveillance video behaviour profiling and anomaly detection. Proc. SPIE, 7486, 74860E.

[5] Hu, D.H.; Yang, Q (2008), Concurrent and interleaving goal and activity recognition. In Proceedings of the National Conference on Artificial Intelligence, Chicago, IL, USA, pp. 1363-1368

[6] Lui, Y.; Beveridge, J.R.; Kirby, M (2010). Action classification on product manifolds. In Proceedings of IEEE Computer Society Conference on Computer Vision and Pattern Recognition, San Francisco, CA, USA, pp. 833-839.

[7] Lui, Y (2012). Advances in Matrix Manifolds for Computer Vision. Image Vision Comput, 30, 380-388

[8] Anice Jahanjoo, Marjan Naderan and Mohammad Javad Rashti (2020), Detection and multiclass classification of falling in elderly people by deep belief network algorithms, Journal of Ambient Intelligence and Humanized Computing.

[9] Shannon, Claude E (1948). A Mathematical Theory of Communication. Bell System Technical Journal. 27(3), 379-423.

[10] Teng Li, Huan Chang, Meng Wang, Bingbing Ni, Richang Hong, and Shuicheng Yan (2015), "Crowded Scene Analysis: A Survey", IEEE Transactions on circuits and systems for video technology, 25(3)

[11] O. Popoola and K. Wang (2012), Video-based abnormal human be-havior recognition -a review Systems, Man, and Cybernet-ics, Part C: Applications and Reviews. IEEE Transactions on , 42(6), 865-878

[12] G. J. Burghouts, V. P. Slingerland, H. ten R.J.M, H. den R.J.M, and K. Schutte (2014), Complex threat detection: Learning vs. rules, using a hierarchy of features, in 11th IEEE International Conference on Advanced Video and Signal Based Surveil lance. IEEE, pp. $375-380$. 
Hoang Manh Ha, Vo Quoc Thong,... - Volume 2- Issue 4-2020, p.363-372.

[13] H. Nallaivarothayan, C. Fookes, S. Denman, and S. Sridharan (2014), An mrf based abnormal event detection approach using motion and appearance features, in 11th IEEE International Confer-ence on Advanced Video and Signal Based Surveil lance. IEEE, pp. 343-348

[14] Y. Benabbas, N. Ihaddadene, and C. Djeraba (2011), Motion pattern extraction and event detection for automatic visual surveillance, Journal on Image and Video Processing, vol. 7, pp. $1-15$.

[15] C. Piciarelli, C. Micheloni, and G. L. Foresti (2008), Trajectory-based anomalous event detection. IEEE Trans. Circu its Syst. Video Techn. , 18(11), 1544-1554.

[16]B. Antic and B. Ommer (2011), Video parsing for abnormality detection. IEEE International Conference on Computer Vision, ICCV 2011, pp. 2415-2422.

[17] A. Adam, E. Rivlin, I. Shimshoni, and D. Reinitz (2008), Robust real-time unusual event detection using multiple fixed-location monitors. IEEE Transactions on Pattern Analysis and Machine Intel ligence, 30(3), 555-560.

[18] M. Roshtkhari and M. Levine (2013), Online dominant and anomalous behavior detection in videos, in Computer Vision and Pattern Recognition (CVPR), 2013 IEEE Conference on, pp. 2611-2618.

[19] V. Mahadevan, W. Li, V. Bhalodia, and N. Vasconcelos (2010), Anomaly detection in crowded scenes, in IEEE Conference on Computer Vision and Pattern Recognition (CVPR), pp. 1975-1981 